\title{
Digital Marketing*
}

\author{
Jerry Wind $^{* *}$, Vijay Mahajan ${ }^{* * *}$
}

\begin{abstract}
The digital revolution has shaken marketing to its core with consumers being offered greater price transparency and often even the chance to dictate the price.

What does pricing mean in a world in which customers propose their own prices (as at priceline.com) or buyers and sellers haggle independently in auctions (as at e-Bay)?

The most significant changes in the digital marketing show the emergence of 'cyber consumers', the cyber business-to-business world and the changing reality of an increasingly complex, dynamic and global markets.
\end{abstract}

Keywords: Digital Revolution; Digital Marketing; Global Markets; Price; Global Competition

\section{Introduction}

The digital revolution has shaken marketing to its core. What does pricing mean in a world in which customers propose their own prices (as at priceline.com) or buyers and sellers haggle independently in auctions (as at e-Bay and many business-to-business auction sites)? Are brands more powerful or less powerful as we move closer to perfect information? What does marketing research mean when companies can track every click of the decision making process? How can they shift from broadcast communications to interactive communication that provides information while educating, entertaining and ultimately persuading the consumer?

As Mohanbir Sawhney pointed out our metaphors for marketing may need to shift. We talk now about marketing as if it were a hunt - 'targeting customers'. We may need to shift to more of a gardening metaphor - 'nurturing ecosystems'. This interactive gardening metaphor is consistent with the digital revolution as articulated by Nicolas Negroponte in his book Being Digital. According to Negroponte, the difference between a television screen and a computer screen becomes one of mere size. And formerly 'mass' media evolves into a personalised two-way street of communication. Information is no longer 'pushed' at consumers.

\footnotetext{
* Adapted from 'Digital Marketing', European Business Forum 'EBF', www.ebfonline.com, n. 1, Spring 2000

** Professor of Marketing, The Wharton School (windj@wharton.upenn.edu)

*** Professor of Marketing, University of Texas-Austin (vijay.mahajan@ mccombs.utexas.edu)
} 
Instead, people or their digital agents pull and help create the specific information they need.

These developments are not unique to the US dot com culture. European consumers, not unlike the consumers in any other culture are empowered by the Internet which creates, despite the attempts of some governments, a truly borderless economy. No business, regardless of its geographical location, is thus immune from the impact of the web revolution and the need to re-examine its fundamental concepts, business models and mode of operations.

\section{The New Realities of Marketing in the Global Digital World}

Among the most significant changes in the business landscape are the emergence of 'cyber consumers' and the cyber business-to-business world, the changing reality of an increasingly complex, dynamic and chaotic world, and the waves of entrepreneurship and innovation and their associated new business models.

\subsection{The Emerging Cyber Consumer}

Technology changes people. Digital technology is changing the way consumers relate to products and markets. It is not just our computers that are being reprogrammed; it is customers themselves. The cyber consumer, as portrayed in Exhibit one, clearly illustrates the need for new marketing approaches to meet their changing needs.

These cyber consumers expect to be able to customise everything - from the products and services they buy, the information they seek, to the price they are willing to pay. They want to be able to engage the producer in the kind of tussle that used to characterise the street bazaar.

Consumers can now sort products based on any desired attribute, price, nutritional value, functionality, etc, or combination of attributes such as price value. They can easily obtain third party endorsements and evaluations including tapping the experience of other users. Empowered by digital technology, customers are unforgiving. Pity the poor company that fails to see this or refuses to play by their rules.

Cyber consumers, though, are not homogeneous. They vary greatly with respect to the degree to which they rely on the Internet. The reliance on the Internet varies by domain (financial services vs food shopping vs automobiles, etc.) and the characteristic of the consumers, especially their 'natural comfort' with the Internet.

\subsection{The Cyber Business-to-Business World}

Whereas most media attention has been on consumers' usage of the Internet and on the new e-commerce start-ups that are aimed at the consumer market, most of the Internet based activities and revenues have been generated in the business-tobusiness world. Indeed, Forester Research Inc estimates current business-tobusiness e-commerce to be five times that of consumer e-commerce and to grow to 10 times by 2003.

The organisational buyer is not only a cyber consumer in his or her personal life, but is supported by a rich and growing Internet and intranet based infrastructure 
that facilitates improved efficiency, effectiveness and innovative new ways of performing traditional corporate roles.

The emergence of increasing numbers of 'virtual marketplaces' - bulletin boards and especially auction-oriented sites (such as Fastparts.com for electronics, PlasticsNet.com for plastics, Metals.com for steel and numerous other sites for almost all industrial products and services) that are bringing together buyers and sellers - are changing the nature of the business-to-business activities.

Companies such as BizBots are developing a real time 24 by seven ( 24 hours a day, seven days a week) automated market of markets that uses 'agents' (or Bots $\mathrm{S} / \mathrm{W}$ ) that link multiple sites in any given business sector (such as chemicals and financial services). These provide full transparency for buyers and sellers on all the information they require to make 'optimal' product/service decisions.

\subsection{A Complex, Dynamic and Chaotic World}

The half-life of change is being compressed. By 2003 Internet users are expected to grow from 130 million today to at least 350 million worldwide users, split between North America - 35\%, Europe - 30\%, Asia Pacific - 21\%, South America $9 \%$ and the rest of the world about 5\%. This phenomenal growth is expected, according to some forecasts, to reach 500 million by 2003 and over 1 billion by 2008. To appreciate the magnitude of this growth, telephone users reached the 1 billion mark only in 1999. The associated e-commerce revenues are expected to grow from close to $\$ 100$ billion in 1999 to over $\$ 1200$ billion in 2003. This expected growth is fuelled by the expected developments of information appliance - access to the Internet via TV, freeing it from the traditional computer keyboard based access. Other modes of access including cellular phones, pagers and other devices that can be used in homes, cars and virtually anywhere are expected to increase the ease of access and pervasiveness of the Internet.

New business models are emerging as rapidly as IPOs by 'dot com' companies. The models encompass $\mathrm{B}$ to $\mathrm{B}, \mathrm{B}$ to $\mathrm{C}, \mathrm{C}$ to $\mathrm{B}$ and $\mathrm{C}$ to $\mathrm{C}$ and are typically based on rapidly building market share and leveraging knowledge. This is in sharp contrast to old models that have focused on profits and returns and leveraging physical assets. The new business models are designed to embrace an unpredictable future by redefining the firm's relationship with its customers and suppliers and by creating new revenue streams.

A unique aspect of the new business models is the ability to patent them as business methods patents. Priceline.com for example is based on a number of patents. These patents are part of the assets of Walker Digital which in mid 1999 included 12 business methods patents and 240 pending ones. These business process patents are an extension of the traditional process patents and are based on new ways of doing business - such as the business model of priceline.com.

Given the eventual need of all companies to provide economic value to their customers, shareholders, and other stakeholders, some of the key management challenges facing companies today are fundamentally marketing challenges. It starts with a need to determine the right metrics for value creation and the valuation of e-business and proceeds with a need to rethink and reinvent the vision, objectives, strategies and organisational architecture based on a reinvented marketing paradigm. 


\section{The New Rules of Marketing in a Digital Age}

These new realities of the business environment have led a number of authors to develop new rules for the new economy. While some of these 'rules' encompass some marketing perspectives companies need to focus more explicitly on a new set of 'principles' for marketing in a digital age. Although these 'rules', as summarised in Exhibit two (TOLTA), are still emerging, they are beginning to shape the new discipline of marketing.

In reviewing these rules it is important to note that while the advances in technology make these rules viable strategies, the success of such strategies ultimately depends on consumer acceptability. Will everyone want, for example, a one-on-one relationship with a company? How are consumers going to deal with the clutter resulting from every company's desire to develop a one-to-one relationship with them?

\subsection{Target Segments of One and Create Virtual Communities}

Companies need to move from mass markets to segments of one. As the name 'segment of one' suggests, companies initially saw these individuals as micro segments. They used database marketing to tailor messages and products to these tiny segments, just as they did with broader customer segments. The concept is now evolving to the point where companies are using a more interactive process and advanced data mining and related techniques to target, attract and retain individual customers as 'partners' in the process. Collaborative filtering techniques, for example, as applied by Firefly Network, allow personalized recommendations to each consumer by comparing their preferences with those of others with similar profiles.

Managers need to determine how to design this process of interaction rather than focusing only on designing specific products and communications. They need to develop effective strategies for giving customers options without overwhelming them. Companies need to suggest possible products without painting customers into a box.

This one-to-one strategy is based on (a) IT systems, such as the one offered by Broad Vision, that deliver personalised information directly to the customer thus allowing Internet based one-to-one marketing and (b) on the recognition that strong customer relationships and management are the key to success. One of the more advanced financial service companies, for example, is creating a large interactive database and decision support system (DSS) that includes, in addition to a real time depository of all the customers activities with the company, the capability to allow continuous dialogue with each customer. Customers who apply for a loan may get a note about the status of the loan application when they use an ATM, look at their statement, or have any other point of contact with the company. The data base, which is augmented with external data including Internet usage, offers the potential for targeting individual customers and developing and offering each customer the products and services that will maximise his or her lifetime value to the firm.

A unique aspect of digital marketing is the ability to create virtual communities for consumers who share a common interest. Amazon.com, for example has been most effective in creating chat rooms on various topics. Virtual Vineyards created a community of consumers interested in finding out about and buying wines. Virtual 
communities center on personal and/or professional relationships and/or transactions and goes beyond the special topics offered by specialised publications to create interactive communities.

Designing and managing a virtual community strategy to complement the 'one on one' segmentation strategy is a must. Such a strategy can and should encompass not only consumers but also suppliers and other stakeholders. Communicating with investors and security analysts, for example, can be greatly facilitated by creating vehicles for interactive communication. In all such virtual community initiatives one can consider planned interventions through the introduction of experts and events around which to mobilise the 'community'.

\subsection{Design for Customer-Led Positioning}

The digital environment can be infinitely manipulated by consumers. While it would be impossible for customers to redesign a physical retail store based on the product attributes they seek, this is quite easy online. With a few mouse clicks on a service such as Peapod's online grocery shopping, the entire store can be reconfigured based on fat content or calories or price. How do companies then position their products in an environment in which customers decide what factors are important and can obtain almost perfect information instantaneously on the performance of the product and its competitors? How do companies position a product when consumer chat rooms and other interactions allow for real time global flow of 'word of mouth' communication? And how can companies take advantage of the community building power of the Internet? Are sites such as Talkway.com precursors to company designed Internet communities that change the dynamics of relationships between the company and its customers and prospects? This may allow the company to decide on a positioning built on customer involvement and relationships while leaving the specific product and service benefits and attributes to a co-determination by the customers and the firms.

When a customer purchases a product from the alliance of the auction powerhouse, Sotheby's and the Internet powerhouse, Amazon.com it is the credibility and positioning of both and the characteristics of the auctioned product, rather than the specifics of the product alone that determines the positioning of and attractiveness of the auctioned item.

When considering a positioning for the changing global digital world it is critical to focus not just on the benefits of the products offered by the firm (and its immediate competitors) but on the benefits sought from the entire range of products and services considered by the consumer (and accessible via the net). For example, effective positioning for a pharmaceutical product is not just the efficacy, safety, convenience and price of the given drug vs its direct competitors, but the benefits sought by consumers who are increasingly concerned about wellness. Increasingly, health sites offer in addition to information and educational material, consultation with a physician (see Cyberdocs.com for example), information on clinical trials, chat groups, filling of prescriptions, (Drugstore.com or PlanetRx.com) and maintenance of health records.

Effective positioning and differentiation increasingly requires focus on 'affect' and emotional ties between the consumer and the brand and the firm offering the products and services. This emotional appeal that was once used primarily in marketing fashion and perfumes is being applied increasingly to hard goods such 
as computers (for example, the 'think different' positioning of Apple's iMac and iBook) and automobiles (for example, as Mercedes-Benz's new positioning as an American treasure).

\subsection{Expand the Role of Branding in the Global Portfolio}

By putting more 'perfect' information in the hands of consumers, digital technology might be expected to undermine the power of brands. Actually, some evidence suggests that branding has become more important. With many options to choose from and less personal relationships online, customers may turn to trusted and trustworthy brands as an indication of more intangible qualities. Nike's brand still has power in this environment because it conveys an image about the purchaser. But an airline brand that relies on low prices and on time performance may be eroded because the information it conveys about budget prices and on-time service can be determined empirically through easily conducted information searches.

The development of brands such as Amazon.com, Yahoo, e-bay, e-trade and other Internet brands can occur much more rapidly with the new technology. But, with the possible exception of 'pure play' Internet brands, all brands should focus on both their meaning and value in both Internet and non-Internet context.

Since digital technology transcends national borders, companies need to pay more attention to the development of global brands. To what extent can brands be globalised and to what extent should companies develop a portfolio of global, regional and local brands? This is especially critical given the heterogeneity of all markets, the growing importance of globalisation, the trend toward M\&A and strategic alliances with other national, regional or global firms which often have their own brands, and the enormous potential of the emerging economies. Some $86 \%$ of the world population is in nations with GNP per capita under $\$ 10,000$. These emerging markets are quite heterogeneous and operate under different principles. If focused on segments other than the one receptive to global brands and positioning, companies cannot simply transfer their US brands, marketing strategies and business models to Islamic or Asian markets, for example. The brands need to be shaped and tailored to carry across different parts of the world, or new brands need to be created.

\subsection{Leverage Consumers as Co-Producers through Customerisation}

From computers to jeans to golf clubs to glasses to greeting cards, companies are moving R\&D out of the labs and into the hands of customers. Instead of the traditional process of new product development, companies need to set up creative mass customisation platforms which allow consumers to design their own products and services.

This new emphasis on customisation means companies need to be careful in presenting customers with options without overwhelming them while assuring that the selected design will function effectively and safely. They also need to be careful they don't overwhelm their own capabilities to execute on what they offer. They need to use the technology to streamline the process of customisation. A hybrid solution is called for in which the company determines the right balance between off-the-shelf options and customisation. 
The advances in flexible manufacturing, information technology, and consumer desire for variety and customisation have led to the emerging trend toward mass customisation, the creation of sense and respond organisations, and more recently customerisation. Mass customisation has drastically changed manufacturing. Its extension to the sense and respond concept is based on electronically sensing customers' needs in real time and using the electronic connection and shared infrastructures to respond to these needs.

Customerisation is taking this concept further by combining the operationally driven mass customisation with customised marketing and empowering the consumers to design the products and service offering of their choice. In contrast to mass customisation, customerisation does not require a lot of prior information about the customer, nor does it require the firm to have its own manufacturing functions. In effect, customerisation redefines the relationship between the firm and its customers. It empowers the customer to design the product and service while the firm 'rents' out to the customer its manufacturing logistics and other resources. The difference between the mass customisation and customerisation is outlined in Exhibit three (TOLTA).

Garden.com is an example of effective customerisation. Customers can design the garden of their choice on their computer, customising the gardens to the local climate. They can select from over 16,000 products and can try out various landscaping options before deciding. Throughout the process they can access any information related to gardening they want and they can order all the products they need with a click of the mouse. Garden.com coordinates the supply chain for the selected products for over 50 suppliers and orchestrates the delivery. It does not own any of the nurseries or transportation vehicles.

While the introduction of a customerisation perspective has enormous implications for the new product development process of the firm, other fundamental changes can not be ignored, such as:

- Product design which assures product updates. Given the rapid technological changes and obsolescence, an increasing number of companies design their products to allow automatic update and upgrade. Nokia for example designs its line of cellular phones with this option and so does Bernina, the premier sewing machine producer.

- Speed of development. As product life cycles shrink, an increasing number of firms focus on innovative concurrent NPD processes, customer involvement in the design process and other approaches for shorter development cycles.

- Concurrent development of the product, process, the associated service offerings and the business model. R\&D should focus not only on the physical product but on the entire product and service offerings, its development and logistic process and its associated business model.

\subsection{Use Creative Pricing in the Priceline.com World}

Digital technology is shifting the pricing power, from companies to customers. The most extreme customer-driven pricing is Priceline.com, in which the customer proposes prices for an airline trip, hotel or even cars and the company decides whether to accept them. 
The surprising thing about the Internet is that, while it facilitates the creation of buying groups (eg Mercata.com) buyer agents services (e.g. BizBuyer.com), provides comparative price information (e.g. CompareNet.com) or facilitates on line auctions (eg eBay or OnSale) customers are sometimes willing to pay a premium for ease of shopping or for the value of customisation.

Companies also are creating value-based pricing so that when the customer wins, the company wins. The company partners with the customer to make the customer more profitable. Then, the value that is created is shared between the company and the customer. For example, Hewlett-Packard is using value-based pricing in a $\$ 500$ million contract to supply computer equipment to Qwest Communications Intl. Qwest pays only based on the revenues it generates and if it achieves its objectives HP will get $\$ 1$ billion in three years. In addition, as part of this arrangement, Qwest will buy $95 \%$ of its Windows computers and $75 \%$ of its Unix servers from HP.

\subsection{Create Anytime-Anyplace Distribution and Integrated Supply Chain}

One of the major impacts of the Internet has been on the disintermediation of the increasing number of industries. The 'Brick and Mortar' distributors and retailers such as Barnes \& Noble face a new reality forced upon them by Amazon.com, while giants such as Merrill Lynch have to reinvent their business strategies in light of the enormous growth of the e-business of CharlesSchwab.com, e-trade, AmeriTrade, and others.

More recently, however, attention has been given to the rise of the infomediaries who link diverse customers to diverse producers. Exchange type firms such as Total Transportation Exchange or Adauction.com provide valuable benefits to its users and create new business models and modes of operations.

An innovative way of distributing and promoting products and services is the Internet version of the traditional sampling which under the name viral marketing offers free service with the hope that it will capture the attention of prospective customers, lead to trial, loyalty and word of mouth 'buzz'. The free e-mail service Hotmail, for example, was one of the pioneers of this type of distribution and was sold to Microsoft for $\$ 400$ million.

Companies need to redesign their supply chains and find the right balance between digital and material distribution. For physical products such as wine or flowers or books, the problem can be complex because the digital component is only part of the equation. It must be joined to a physical distribution network such as Fed Ex or UPS or creates its own. The use of technology on the business-tobusiness side can help integrate the supply networks, reduce product inventory, streamline processes and as a result cut cost and speed up the development, manufacturing and delivery of products and services.

\subsection{Redesign Advertising as Interactive and Integrated Marketing Communication Education and Entertainment}

Advertising and other marketing communications were shaped by the mass media. The new interactive media created by digital technology is spawning a very different type of communication with customers. It is more addressable and responsive, moving from broadcast media to interactive media. This shift and especially the advances in technology that are lowering costs of the technology, 
providing Internet access through television set-top boxes and introduces interactivity, challenges the current 'troika' of the advertising industry-advertisers, agencies and media - and redefines direct marketing.

The content of the communications is also changing. Customers are no longer the passive recipients of ads and commercial information, but are active participants in an interactive 'edutainment' process seeking the tools to learn about the products and services (education) while being entertained and inspired. Media advertising, Internet communication, public relations, packaging, customer service and any other point of contact between the firm and its customers and prospects has to be integrated.

The more captivating and engaging a website's design, the more likely customers are to spend time on the site and develop relationships with the company. An interactive design which is consistent with and reinforcing of the design elements of the other marketing activities of the firm is a must. This has to be augmented with a network of strategic alliances and marketing agreements to assure access to the site from other Internet access points. Consider for example the aggressive strategic alliances of Bank One that include relationships with AOL and Excite (including co-branding), Broadcast.com, CarsDirect.com (to select, finance and purchase cars over the Internet), CFN, Cnet, CNN, Cybercast, eBay, GeoCities, inane, iVillage, Lifeserve, Microsoft Network, Peapod, SportsLive, ValueAmerica, WebTV, Yahoo and others.

\subsection{Reinvent Marketing Research As Knowledge Creation And Dissemination}

While the costs of gathering data drop significantly with the addition of digital to traditional sources (face-to-face interviews, mail or telephone interviews), the avalanche of new and old information creates new costs and requires new competencies for the development and use of effective data mining tools. Data mining and other forms of analysis are the key to a firm's ability to target its customers and prospects based on their lifetime value to the firm as well as the basis for identifying and meeting the customer's needs and wants.

The technology benefits marketing by making tacit knowledge more accessible. Some of the most important knowledge about customers is tacit knowledge, such as their shopping patterns, interest and activities profiles, payment transactions and chatroom discussions. These can all be captured online. But companies need to find ways to get customers to part with the knowledge and they also need to be able to capture and use the knowledge once they obtain it.

While data mining is gaining in popularity, the rest of marketing research cannot just stay as it is. A major reinvention of the marketing research function is called for by focusing on at least four areas:

- diagnose problems rather than just test solutions;

- increase speed and efficiency by using new information technology;

- take an integrated approach;

- expand the strategic impact of marketing.

Key aspects of the new approaches to marketing research offered by the new technology are the opportunities it offers to the firm, and to new type Internet marketing research firms, to collect information that is initiated and generated by customers and other stakeholders. In this context intriguing opportunities are 
offered by applications such as ThirdVoice.com that lets anyone attach post-it type notes throughout any website. The notes do not alter the underlying site but allow active interaction among all who are interacting with the site.

To fully capitalise on the opportunities the new marketing research offers the firm, often requires a reinvention of the marketing research function and repositioning it as the knowledge engine of the firm.

\subsection{Use Adaptive Experimentation}

Adaptive experimentation has always been a powerful philosophy and methodology allowing learning while doing and thus 'optimising' the strategy over time.

The major benefits of adaptive experimentation - ability to continuously learn, added incentive to develop and test innovative strategies, making it harder for the competition to figure out what your strategy is and creating a culture of experimentation and learning - are even more critical in the changing and turbulent digital marketing environment. At the same time the advances in technology allow easier and better ways to design and implement 'master experiments' across various products, marketing strategy elements and geographies. Whereas adaptive experiments require the identification of the key marketing variables to be tested - advertising level and message, sales force size and approach, pricing options, etc. A somewhat less structured approach, but in the spirit of continuous experimentation has been developed by firms such as Seiko. Seiko develops over 2500 new watch designs a year and introduces them in a number of 'test markets' the successful designs are further developed, tested again and then launched in the target markets.

\subsection{Redesign the Strategy Process and Supporting Organisational Architecture}

Key to success in the changing business environment is the understanding of the changes, their drivers, their interdependencies and consequences and their summary in meaningful scenarios. Having established the scenarios, the focus of management should be on establishing:

- a vision which combines the company's own aspirations with the type of firm that will succeed in the changing environment and which should incorporate an explicit reference to the firm's vision for its e-business and the role of e-business in enhancing and achieving its overall corporate vision;

- measurable stretch objectives encompassing all key performance measures such as maximizing the lifetime value from target customer segments or minimizing the time to peak sales. Explicit objectives regarding the ebusiness aspects of the business, such as share of target market or revenue from e-business or even objectives to spin off a high market cap e-business is essential;

- having established the vision and stretch objectives the key is the development of creative strategies. This requires, at the minimum:

- a re-examination of the business model and value proposition;

- innovative strategies reflecting the ten new rules of marketing;

- having established the e-business strategy and the other strategies of the firm, including strategic alliances, attention should be given to the 
determination of the best organisational design. Companies are experimenting today with a variety of organisational designs including:

- incorporating e-business throughout the organisation (for example, Prudential California Realty);

- creating e-business subsidiaries (for example, MagazineOutlet.com a subsidiary of Newsweek Services Inc.);

- creating separate e-business subsidiaries with the objective of spinning them off as separate online activities (for example, BarnesandNoble.com which was spun off by Barnes and Noble as a separate publishing company);

- investing in or merging with Internet companies (for example, Pet Smart, a pet supply chain, invested in PetJungle.com or Rite Aid investment in Drugstore.com);

- shifting the entire business to the Web (for example, Latham [real estate] auctioneers changed their business to a Web only operation called Homebid.com);

- the final set of decisions involves the redesign of the supporting organisational architecture assuring that it capitalises on the advances in Internet and other information technology. This redesign should include the seven interrelated components of the organisational architecture:

- the organisational culture and values;

- the organisational structure;

- the value creation processes including those for knowledge creation, dissemination and use;

- the people and their required competencies;

- the required technology and especially the IT infrastructure;

- the required resources;

- the performance measures and incentives

\section{Conclusions}

As the digital revolution has taken place, companies have tended to look at the technology itself while paying too little attention to its implications for marketing. Yet as the world shifts from physical to virtual, as value moves from the greasy gears and hard steel of the industrial age to the high concepts of a knowledge economy- what could be more important to companies than the relationships with customers and other external constituencies, knowing their needs and how to communicate with them and meeting their changing needs and building sustainable relationship and loyalty?

The reinvention of marketing requires a re-examination of all the concepts, methods and practices of marketing to assure their appropriateness for the changing global digital environment. This poses an enormous challenge both to the academic community and the business practitioners. For academics this poses the most significant challenge of changing their research agenda. A change that requires a shift toward a multi-disciplinary cross-functional perspective based on a thorough understanding of the power and pervasive impact of the Internet and related advances in information technology. The challenge for the emerging research agenda is how to avoid becoming 'reporters' of current advanced management practices and 
build on the methodological and traditional strengths of the discipline to provide management with useful new conceptual and methodological tools to cope with and succeed in the changing global digital world.

The recommendations in this article are not just motivated by a parochial interest in seeing marketing elevated in the organisation, but by a recognition that marketing and especially as it is shaped by the new marketing paradigm, is a central philosophy, approach and set of activities that should be adapted by all executives. If we have great technology that is not focused on the market, what value will it provide? What advantages will it create? By understanding the new global digital reality and focusing on the new rules of marketing, marketing professionals can begin to reclaim some of their lost ground and corporate executives can increase their likelihood of success in the turbulent, chaotic, changing global digital environment. 